\title{
SAND99-1755J
}

\section{Origin of compact triangular islands in metal-on-metal growth}

\author{
Staffan Ovesson, ${ }^{a}$ Alexander Bogicevic, ${ }^{b}$ and Bengt I. Lundqvist ${ }^{a}$ \\ a Department of Applied Physics, Chalmers University of Technology and Göteborg University, S-412 96 Göteborg, Sweden \\ ${ }^{b}$ Department of Surface and Interface Sciences, Sandia National Laboratories, Albuquerque, NM 87185-1413, USA
}

(May 28, 1999)

\begin{abstract}
The microscopic origin of compact triangular islands on close-packed surfaces is identified using kinetic Monte Carlo simulations with energy barriers obtained from density-functional calculations. In contrast to earlier accounts, corner diffusion anisotropy is found to control the shape of compact islands at intermediate temperatures. We rationalize the correlation between the orientation of dendrites grown at low temperatures and triangular islands grown at higher temperatures, and explain why in some systems dendrites grow fat before turning compact.

PACS number(s): 68.55.-a, 66.30.Fq, 68.35.Fx, 68.60.-p
\end{abstract}

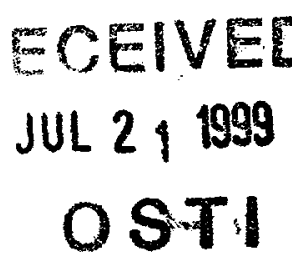

Epitaxial growth of metals is governed by a handful of elementary atomic diffusion processes, where motion of atoms along and across clusters of adsorbed atoms is inherently different from diffusion on plain terraces $[1,2]$. In particular, sites of low coordination and symmetry do not only always influence growth, but very often directly control it [3]. For example, diffusion processes at kinks and corners define the shape of atomic aggregates [3], the mechanism by which these migrate across the surface $[3,4]$, and the very growth mode itself [5]. Here low-symmetry diffusion processes are shown to have additional important roles, and three outstanding morphology issues in epitaxial growth are resolved.

In a seminal scanning-tunneling-microscopy (STM) study of homoepitaxial growth of Pt(111), Michely and coworkers [6] observed several beautiful transitions in surface morphology upon increasing the substrate temperature $T$. Focusing on compact island shapes, triangular islands bounded by A-steps at $400 \mathrm{~K}$ were observed to become inverted at $640 \mathrm{~K}$, bounded by B-steps (see Fig. 1 for clarification of step types). A simple explanation for triangular islands was proposed by Michely et al. [6]: growing islands should advance faster perpendicular to steps with lower adatom mobility. Accordingly, islands would tend to become triangular, with the faster growing steps disappearing. The shape transition from A- to Bstep-bounded islands was attributed to a cross-over in the rate for diffusion of atoms along the two types of steps. Specifically, an anisotropy in both activation barriers and prefactors for edge diffusion was proposed to induce a cross-over in the relative growth speed of these steps, inverting the triangular islands. Density-functional calculations for $\mathrm{Al} / \mathrm{Al}(111)$ by Stumpf and Scheffler [7] indeed do show anisotropic edge diffusion, with barriers for diffusion along $\mathrm{A}$ - and B-steps of 0.32 and $0.39-0.42 \mathrm{eV}$, respectively. With the additional (and vaguely motivated) assumption of a 100 times larger prefactor for edge diffusion along $\mathrm{B}$-steps, the shape transition, experimentally observed for $\mathrm{Pt} / \mathrm{Pt}(111)$ [6], could be qualitatively reproduced in kinetic Monte Carlo (KMC) simulations of Al(111) growth [8].
By rescaling semi-empirically calculated energy barriers for Pt/Pt(111), Jacobsen and coworkers [9] have been able to find a parameter set that reproduces the experimentally observed transitions, but with the governing anisotropies instead lying in the barriers for kink breaking and corner crossing. Similar conclusions have been drawn by Liu and coworkers in another KMC study [10].

As it turns out, however, these different rationalizations have been made with respect to contaminated specimens. Recent STM experiments by Kalff, Comsa, and Michely [11] have revealed that the triangular orientation observed at low temperatures is an effect of carbon monoxide sticking preferentially to A-steps. In the renewed experiment, with greatly reduced amounts of contaminants, only triangular islands bounded by B-steps are observed, even at the lower temperature.

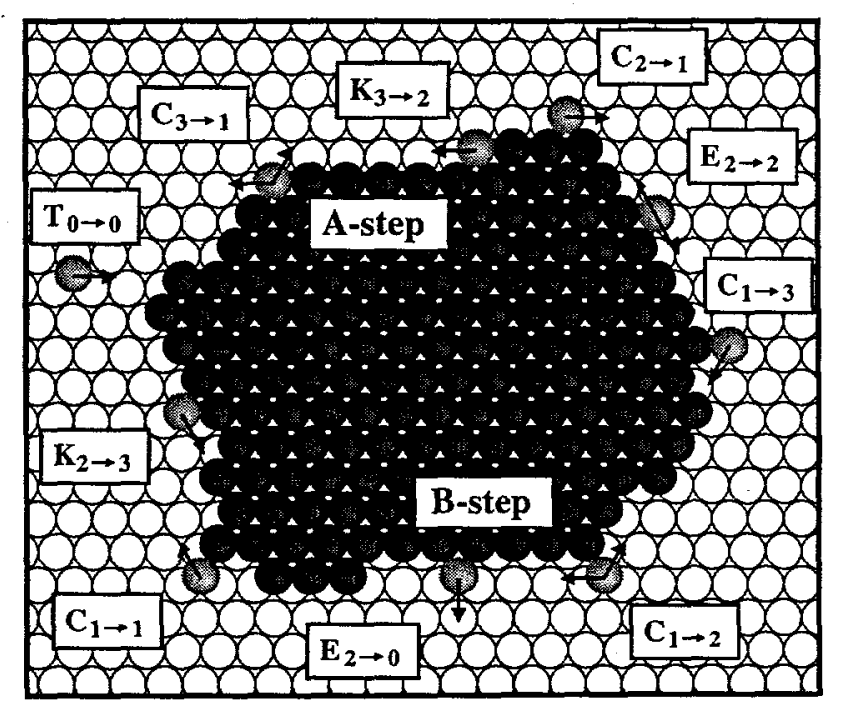

FIG. 1. Illustration of elementary diffusion processes. Each process is characterized by a letter ( $\mathrm{T}$ for terrace, $\mathrm{E}$ for edge, $\mathrm{K}$ for kink, and $\mathrm{C}$ for corner) and a subscript that indicates the number of in-layer nearest neighbors before and after the jump. The processes can take place at both A-steps, with a $\{100\}$ microfacet, and B-steps with a $\{111\}$ microfacet. 


\section{DISCLAIMER}

This report was prepared as an account of work sponsored by an agency of the United States Government. Neither the United States Government nor any agency thereof, nor any of their employees, make any warranty, express or implied, or assumes any legal liability or responsibility for the accuracy, completeness, or usefulness of any information, apparatus, product, or process disclosed, or represents that its use would not infringe privately owned rights. Reference herein to any specific commercial product, process, or service by trade name, trademark, manufacturer, or otherwise does not necessarily constitute or imply its endorsement, recommendation, or favoring by the United States Government or any agency thereof. The views and opinions of authors expressed herein do not necessarily state or reflect those of the United States Government or any agency thereof. 


\section{DISCLAIMER}

Portions of this document may be illegible in electronic image products. Images are produced from the best available original document. 
In this Letter, we demonstrate that, while edge diffusion is necessary for the growth of compact islands, anisotropic edge diffusion is very unlikely to give rise to triangular islands. Instead, the origin of the triangular growth mode is shown to lie in an anisotropy in corner diffusion. Such anisotropy has been indirectly deduced from low-temperature STM studies of several metal-on-metal systems [2], and has recently been calculated from first principles for $\mathrm{Al} / \mathrm{Al}(111)$ [3]. It has also been noted for several other systems in semi-empirical calculations $[9,15]$. These findings indicate that our conclusions are general for a wide class of metal systems.

The method we use is the kinetic Monte Carlo scheme, as introduced by Voter [12]. This simulation technique renders the time and length scales of typical growth experiments accessible to materials theorists by describing elementary stochastic processes (diffusion, deposition, etc.) in terms of reaction rates (energy barriers and prefactors) to avoid explicit calculations of unsuccessful attempts.

Even though $\mathrm{KMC}$ is a powerful technique, as demonstrated for a wide range of metal-on-metal systems $[2,8-10]$, it too has its limitations. The predictive power is limited because of the sensitivity of growth phenomena to relatively small changes in diffusion barriers. KMC simulations have hitherto been performed using rates calculated from model potentials (for exceptions, see below), or inferred from fits to experimental data. Activation energies computed using model potentials are of limited accuracy, and do not seldom differ by a factor of two or more from more accurate values. Part of this discrepancy can be removed by scaling all barriers with a common factor, but the results can, of course, still be very uncertain. Finding a set of barriers that reproduces experimental observations is even more awkward, because the necessity of considering low-symmetry elementary processes in the simulations gives rise to a huge parameter space, which may contain several regions that reproduce experiments fairly well. The accuracy provided by today's first-principles calculations is therefore desired in the determination of activation energies needed as input to $\mathrm{KMC}$ simulations.

In a pioneering study of the $\mathrm{Al} / \mathrm{Al}(111)$ system [7], Stumpf and Scheffler calculated the energy barriers for monomer and edge diffusion from first principles, and later used these (together with semi-empirical barriers) in $\mathrm{KMC}$ simulations [8]. However, epitaxial growth is to a large extent governed by various low-symmetry diffusion processes that must be accounted for properly in KMC simulations. Such low-symmetry processes have recently been mapped out for $\mathrm{Al} / \mathrm{Al}(111)$ from first principles, and shown to directly control shape transitions and the migration of large islands [3].

The guiding principle of this $\mathrm{KMC}$ study is to base our calculations on the post-GGA barriers (GGA corrections to an otherwise LDA-described system) reported in

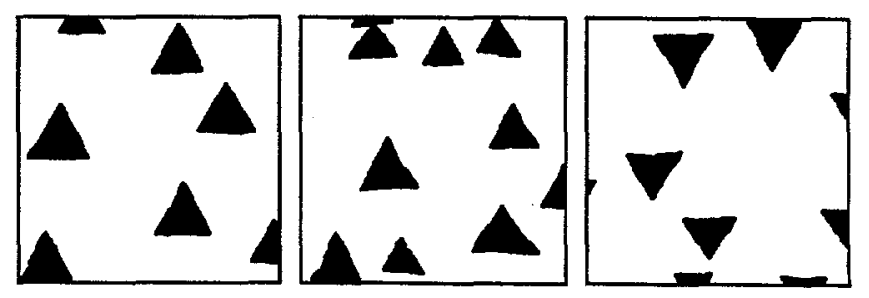

FIG. 2. KMC results for the morphology of the $\mathrm{Al}(111)$ surface at $T=160 \mathrm{~K}, F=0.0018 \mathrm{ML} / \mathrm{s}$. The left-most figure shows an ensemble of islands obtained with first-principles parameters taken from Ref [3]. Reversing the edge diffusion anisotropy has no effect on the orientation of the triangular islands (middle panel). The island orientation changes only when the corner diffusion $\left(\mathrm{C}_{1 \rightarrow 2}\right)$ anisotropy is reversed (right-most panel). In all three figures, the coverage is $0.10 \mathrm{ML}$ and the image size $680 \times 680 \AA^{2}$.

Ref. [3], but to be free to vary any input parameter, one at a time, and in this way test the extent of our conclusions.

The prefactors for all processes are here set to $6 \cdot 10^{12} \mathrm{~s}^{-1}$ (calculated within the harmonic approximation of transition-state theory in Ref. [3]). Since prefactors enter the rates linearly, in contrast to the barriers which enter exponentially, small variations in prefactors are unimportant. For monomer diffusion, however, we use the experimentally determined (through a nucleation theory analysis of island densities) prefactor of $10^{7}$ $s^{-1}[13]$. This induces a higher island density that helps keep down the computational demands. However, the conclusions presented here do not depend on this choice for the monomer prefactor, as rigorously tested by employing the higher prefactor also for monomer diffusion.

The simulations are carried out on an $800 \times 800$ atom close-packed lattice with periodic boundary conditions. The atoms are deposited at random on an initially clean surface, so that the dynamics of nucleation and attachment to islands are fully accounted for. The deposition flux $F$ is set to $0.0018 \mathrm{ML} / \mathrm{s}$ at $160 \mathrm{~K}$ and then adjusted to keep the ratio $D / F$ fixed ( $D$ being the monomer diffusivity), which results in a nearly constant island density.

Effect of corner diffusion anisotropy. - The KMCsimulated surface morphology at $\mathrm{T}=160 \mathrm{~K}$ is shown in Fig. 2. The islands exhibit a sharp triangular form, whose orientation is consistent with the anisotropic-edge-diffusion argument of Refs. $[6,8]$, since here $\Delta E\left(\mathrm{E}_{2 \rightarrow 2}^{\mathrm{A}}\right) / \Delta \mathrm{E}\left(\mathrm{E}_{2 \rightarrow 2}^{\mathrm{B}}\right)=0.31 / 0.26>1$, where $\Delta E$ is the activation energy that enters the simulations. However, reversing or turning off the edge diffusion anisotropy has no effect on the island shape (Fig. 2), despite the calculated anisotropy being stronger here (in terms of diffusion rates) than suggested in Refs. [6,8].

On the other hand, a drastic change occurs when, with all other parameters fixed, the anisotropy in corner diffusion $\left(\mathrm{C}_{1 \rightarrow 2}\right)$ is reversed: the triangles are turned by $60^{\circ}$, exposing only A-steps (Fig. 2). This result can be rationalized by considering the step-edge energies. With the original parameter set, the balance between the barriers 


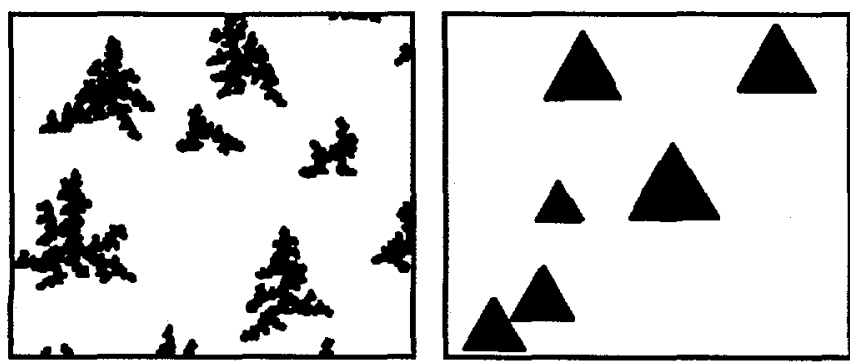

FIG. 3. The island morphology at $80 \mathrm{~K}$ (left) reveals dendrites with the same orientation as triangles grown at $200 \mathrm{~K}$ (right). Note that the islands at $200 \mathrm{~K}$ are nearly identical with those grown at $160 \mathrm{~K}$ (Fig. 2). In both figures, the coverage is $0.10 \mathrm{ML}$ and the image size $680 \times 570 \AA^{2}$.

for corner diffusion and corner crossing $\left(\mathrm{C}_{2 \rightarrow 1}\right)$ along Aand $B$-steps leads to an energy gain of $0.17 \mathrm{eV}$ upon diffusion from a $\mathrm{B}$ - to an A-step. Atoms thus accumulate on A-steps, which then grow faster, and are eventually depleted.

By equalizing the A- and B-step energies, while maintaining the anisotropy in edge diffusion, we obtain hexagonal islands with an edge-length ratio $L_{\mathrm{A}} / L_{\mathrm{B}}$ of $\approx 2$. The orientation obtained with edge diffusion anisotropy only is thus the opposite of the one suggested in Ref. [6], as explained by Jacobsen et al. [9]. Obviously, anisotropic edge diffusion does influence the growth morphology, but at $160 \mathrm{~K}$ the relative population of edge atoms along $\mathrm{A}$ - and B-steps is determined by thermodynamics rather than kinetics. Consequently, the (purely kinetic) effect of anisotropic edge diffusion has saturated, and the shape of the islands is instead governed by corner energetics.

In line with previous findings for other metal systems [14-16], the structures grown at $80 \mathrm{~K}$ are dendrites (fractals growing in three preferential directions) with branches perpendicular to A-steps. This asymmetry has been shown to be governed by the anisotropy in corner diffusion, as well $[2,3]$. Consequently, the orientation of dendrites at low $T$ and triangular islands at high $T$ are correlated, i.e. low- $T$ dendrites and high- $T$ triangles "point" in the same directions (Fig. 3). This relationship, first pointed out by Brune [2], is thus hereby given an explanation in terms of corner diffusion anisotropy.

The suggestion that kink breaking $\left(\mathrm{K}_{3 \rightarrow 2}\right)$ is necessary for growth of sharp triangles [10] is not corroborated in the present study, as turning off this process does not affect the island shape. This result is important for another reason: it shows that our conclusions are not influenced by lack of detailed balance [20], which can easily become a problem and give rise to artificial growth structures as soon as diffusion processes at kinks are allowed. In fact, the growth morphology at $200 \mathrm{~K}$ - well above the activation temperature for kink breaking [3] - is exactly the same as at $160 \mathrm{~K}$ : nearly perfect triangles (Fig. 3).

The fractal to compact island transition. - The transition from irregular fractals to compact islands has been

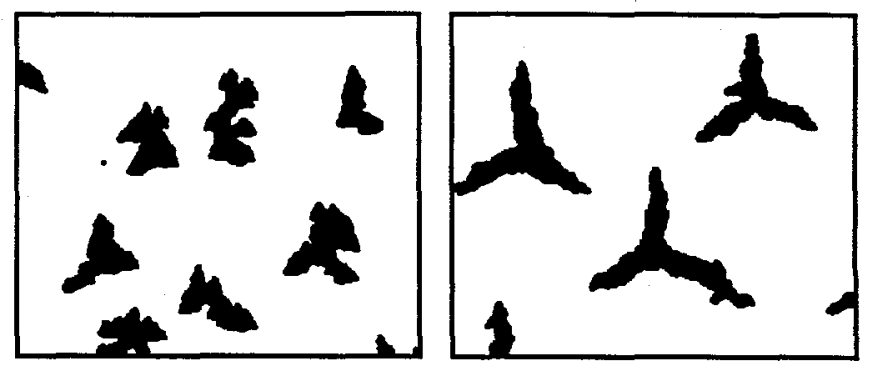

FIG. 4. The simulated island morphology at $120 \mathrm{~K}$. The left figure is the result with parameters taken from Ref [3]. In the right figure, the edge diffusion anisotropy has been reversed. In both figures, the coverage is $0.10 \mathrm{ML}$ and the image size $680 \times 570 \AA^{2}$.

studied experimentally and found to proceed in two ways, depending on material [2]. In some systems, there is a direct transition from thin (about one atom wide) dendrites at low $T$ to compact islands at high $T$, whereas in other systems the transition is smooth with increasingly fatter dendrites turning into compact islands. Here we present an explanation for this interesting phenomenon.

Since at $160 \mathrm{~K}$ the corner energetics overrides any ef fects due to anisotropic edge diffusion, the significance of the latter anisotropy should be more pronounced at lower temperatures, where kinetics is more important. We find this to be true, but in an unexpected way. Just like at $80 \mathrm{~K}$, edge diffusion and corner crossing events are not frequent enough at $120 \mathrm{~K}$ to drive the formation of compact islands. However, the islands grown at $120 \mathrm{~K}$ differ qualitatively from the dendrites obtained at $80 \mathrm{~K}$, where these two processes are basically unactivated. The islands grown at the intermediate temperature $(120 \mathrm{~K})$ are very irregular, semi-compact, and retain no or little anisotropy, see Fig. 4. By reversing the edge diffusion anisotropy at this temperature, we instead get fat dendrites with the same orientation as those obtained at $80 \mathrm{~K}$ (Fig. 4). Our simulations show that an (at least partial) explanation to this phenomenon is that a smooth transition with fat dendrites occurs whenever $\Delta E\left(\mathrm{C}_{1 \rightarrow 2}^{\mathrm{A}}\right)<\Delta \mathrm{E}\left(\mathrm{C}_{1 \rightarrow 2}^{\mathrm{B}}\right)$ and $\Delta E\left(\mathrm{E}_{2 \rightarrow 2}^{\mathrm{A}}\right)<\Delta \mathrm{E}\left(\mathrm{E}_{2 \rightarrow 2}^{\mathrm{B}}\right)$ or vice versa (exchange $A$ for $B$ ). The dendritic character remains when both edge diffusion barriers are set to either of the A- and B-step values, indicating the significance of edge diffusion anisotropy here. These observations can be understood in the following way: The anisotropies in edge diffusion and corner diffusion both tend to generate triangular islands, but depending on the relations between these barriers, they may or may not favor the same orientation. If they do, corner and edge diffusion cooperate in the formation of compact triangles, and the transition from dendrites at low $T$ to triangles at high $T$ is smooth, with edge diffusion making the branches of the islands fatter and fatter until they coalesce to form a triangle. If the two anisotropies counteract (as is the case with the original parameter set), edge diffusion obstructs 
the growth of dendrites, which in turn makes the islands more compact.

This is a delicate point where state-of-the-art DFT calculations for $\mathrm{Al} / \mathrm{Al}(111)$ presently fail to deliver the adequate information, because $\Delta E\left(\mathrm{E}_{2 \rightarrow 2}^{\mathrm{A}}\right)$ is lower than $\Delta E\left(\mathrm{E}_{2 \rightarrow 2}^{\mathrm{B}}\right)$ within the LDA, whereas the opposite holds for post-GGA (note that our main conclusions are independent of such detailed information of barrier values). Nevertheless, Fig. 4 illustrates that experiments could provide a very pictorial test of which exchangecorrelation functional is closer to reality.

At this point, it is important to establish how general these results really are. We argue that our conclusions apply to homoepitaxial growth on any close-packed fcc metal surface. Of course, the results would be different for a system in which the major part of the step-energy difference is due to anisotropy in corner crossing rather than corner diffusion. However, such an anisotropy has never been demonstrated, theoretically or experimentally, while first-principles calculations for $\mathrm{Al} / \mathrm{Al}(111)$ [3] and semi-empirical calculations for other systems [15] show that the anisotropy in corner diffusion is strong (a factor of 2 or more in the barrier). This has also been observed indirectly in low-temperature STM experiments, where in most systems dendrites (rather than random fractals) are observed $[2,11,16,17]$. At temperatures as low as $30 \mathrm{~K}$, this means that the barrier for the slower corner diffusion process typically must be almost twice as high as for the faster one to give rise to anisotropic dendrites. Finally, we note that recent DFT calculations of dimer self-diffusion on $\mathrm{Pt}(111)$ [19]. get qualitatively identical results as first calculated for $\mathrm{Al}(111)$ [18], implying that results for one system might carry over to a wide class of materials. There is thus ample reason to believe that the results presented here are quite general.

In summary, we propose a new mechanism as the origin for the formation of compact triangular islands in epitaxial growth of close-packed metals. Our conclusions are based on $\mathrm{KMC}$ simulations for the $\mathrm{Al} / \mathrm{Al}(111)$ system, with activation barriers determined from DFT calculations [3]. The orientation of the triangular islands is found to be governed by the anisotropy in corner diffusion, which explains the correlation between the orientations of anisotropic dendrites grown at low temperatures and the triangles obtained at higher temperatures. Anisotropic edge diffusion is seen to be important only at intermediate temperatures, where it very strongly affects the transition from dendritic to compact islands.

We thank Harald Brune and Hannes Jónsson for constructive remarks, and Joachim Jacobsen for lending us his KMC code. Sandia is a multiprogram laboratory operated by Sandia Corporation, a Lockheed Martin Company, for the United States Department of Energy under Contract DE-AC04-94AL85000. This work is partially supported by a Laboratory Directed Research and Development project and by the Swedish Research Council for Engineering Sciences (TFR).

[1] G. L. Kellogg, Surf. Sci. Rep. 21, 1 (1994).

[2] H. Brune, Surf. Sci. Rep. 31, 121 (1998).

[3] A. Bogicevic, J. Strömquist, and B. I. Lundqvist, Phys. Rev. Lett. 81637 (1998).

[4] A. Bogicevic, S. Liu, B. I. Lundqvist, and H. I. Metiu, Phys. Rev. B 57, R9459 (1998).

[5] E. Lundgren et al., Phys. Rev. Lett. 82, No. 25 (in print).

[6] T. Michely, M. Hohage, M. Bott, and G. Comsa, Phys. Rev. Lett. 70, 3943 (1993).

[7] R. Stumpf and M. Scheffler, Phys. Rev. Lett. 72, 254 (1994).

[8] C. Ratsch, P. Ruggerone, and M. Scheffler, in Surface Diffusion: Atomistic and Collective Processes, edited by M. C. Tringides (NATO ASI Series B: Physics Vol. 360, Plenum Press, New York 1997).

[9] J. Jacobsen, K. W. Jacobsen, and J. K. Nørskov, Surf. Sci. 359, 37 (1996).

[10] S. Liu, Z. Zhang, G. Comsa, and H. Metiu, Phys. Rev. Lett, 71, 2967 (1993).

[11] M. Kalff, G. Comsa, and T. Michely, Phys. Rev. Lett. 81, 1255 (1998).

[12] A. Voter, Phys. Rev. B 34, 6819 (1986).

[13] J. Barth, H. Brune, B. Fischer, J. Weckesser, and K. Kern, to be published.

[14] H. Brune, C. Romainczyk, H. Röder, and K. Kern, Nature 369, 469 (1994).

[15] H. Brune, H. Röder, K. Bromann, K. Kern, J. Jacobsen, P. Stoltze, K. Jacobsen, and J. Nørskov, Surf. Sci. 349, L115 (1996).

[16] M. Hohage, M. Bott, M. Morgenstern, Z. Zhang, T. Michely, and G. Comsa, Phys. Rev. Lett. 76, 2366 (1996).

[17] B. Fischer, J. Weckesser, J. Barth, H. Brune, and K. Kern, to be published.

[18] A. Bogicevic, P. Hyldgaard, G. Wahnström, and B. I. Lundqvist, Phys. Rev. Lett. 81, 172 (1998).

[19] G. Boisvert and L. J. Lewis, Phys. Rev. B 59, 9846 (1999).

[20] By going around an irregular island and keeping track of the relative binding energy, it is possible to return to the same position at a different binding energy than at the start. This "perpetuum mobile" artifact can easily appear as soon as three-foldly coordinated atoms are allowed to dissociate. 